\title{
CODETERMINANTS AND $q$-SCHUR ALGEBRAS
}

\author{
GERALD CLIFF AND ANNA STOKKE
}

\begin{abstract}
We study codeterminants in the $q$-Schur algebra $S_{q}(n, r)$ and prove that the standard ones form a basis of $S_{q}(n, r)$, using a quantized version of the Désarménien matrix. We find elements of the form $F_{S} 1_{\lambda} E_{T}$ in Lusztig's modified enveloping algebra of $g l(n)$, which, up to powers of $q$, map to the basis of standard codeterminants, where $F_{S} \in U^{-}$and $E_{T} \in U^{+}$are explicitly given products of root vectors, depending on Young tableaux $S$ and $T$.
\end{abstract}

\section{INTRODUCTION}

Let $K$ be an infinite field, and let $n$ and $r$ be positive integers. The classical Schur algebra $S(n, r)$ can be defined as the dual $A(n, r)^{*}$ of the $K$-space of homogeneous polynomials of degree $r$ in $n^{2}$ variables $x_{i j}, 1 \leq i, j \leq n$. Since $A(n, r)$ is a coalgebra, $S(n, r)$ is an associative algebra. The space $A(n, r)$ has a basis of monomials $\left\{x_{I, J}\right\}$, and the corresponding dual basis $\left\{\xi_{I, J}\right\}$ is a basis of $S(n, r)$. J. A. Green [7] defined another basis of $S(n, r)$, consisting of standard codeterminants, each of which is a certain product $\xi_{I, J} \xi_{J, K}$. Green used these codeterminants to give combinatorial proofs of results about Schur algebras, for example that they are quasi-hereditary.

We wish to do the same for the $q$-Schur algebra $S_{q}(n, r)$. This was defined by Parshall-Wang [14] by replacing the commutative polynomial ring in the $n^{2}$ variables $x_{i j}$ by Manin's coordinate ring of quantum $A_{q}(n)$ matrices. Then $A_{q}(n, r)$ is the $r$-th homogeneous part of $A_{q}(n)$, and $S_{q}(n, r)$ is defined to be the dual $A_{q}(n, r)^{*}$. We define quantized codeterminants, following Green's approach as much as possible. Green needed the Désarménien matrix, which has a quantized version due to Stokke [16].

The $q$-Schur algebra was also defined by Beilinson-Lusztig-Macpherson [1] using structure constants which arise from the consideration of flags in vector spaces over a field of $q$ elements, and also by Dipper-James [2], [3] as the commuting ring of a suitable generalized permutation module for the Iwahori-Hecke algebra (of type A.) Using these versions of the $q$-Schur algebra, R. M. Green [9] defined quantized codeterminants, and proved a straightening formula for them, generalizing work of Woodcock [18] for the classical Schur algebra. Our approach for defining codeterminants is much closer to that taken by J.A. Green in the classical setting. We pair off standard codeterminants with standard bideterminants in $A_{q}(n, r)$. We give a Carter-Lusztig basis for the $q$-Weyl module in terms of these codeterminants in Section 6.

This research was supported in part by grants from the Natural Sciences and Engineering Research Council of Canada. 
In [1] (see also [5]) it is shown that the $q$-Schur algebra is a homomorphic image $\theta\left(\dot{U}_{q}\right)$ of a modified quantized enveloping algebra $\dot{U}_{q}$. This $\dot{U}_{q}$ can be obtained from the quantized enveloping algebra $U_{q}$ of the general linear group, using the triangular decomposition $U_{q}=U^{-} U^{0} U^{+}$of $U_{q}$, replacing $U^{0}$ by a direct sum of infinitely many one-dimensional algebras, generated by idempotents $1_{\lambda}$, one for each weight $\lambda$. We show that the standard codeterminants, up to powers of $q$, have the form $\dot{\theta}\left(F_{S} 1_{\lambda} E_{T}\right)$ where $F_{S} \in U^{-}$and $E_{T} \in U^{+}$are explicitly given products of root vectors, depending on Young tableaux $S$ and $T$.

\section{Young tableaux}

A partition of a fixed positive integer $r$ is a $k$-tuple of positive integers $\lambda=$ $\left(\lambda_{1}, \lambda_{2}, \ldots, \lambda_{k}\right)$ with $\lambda_{1} \geq \lambda_{2} \geq \cdots \geq \lambda_{k}$ and $\sum_{i=1}^{k} \lambda_{i}=r$. The Young diagram of shape $\lambda$ consists of $r$ boxes arranged in $k$ left-justified rows with the $i$-th row consisting of $\lambda_{i}$ boxes. One obtains a $\lambda$-tableau $T$ by filling the Young diagram of shape $\lambda$ with entries from a set $\{1,2, \ldots, n\}, n$ a positive integer. Throughout the article, $n$ and $r$ will be fixed, with $\lambda$ a partition of $r$ and $\lambda$-tableaux having entries in $\{1,2, \ldots, n\}$.

Let $T$ be a $\lambda$-tableau. Then $T$ is called standard if the entries in $T$ are weakly increasing across the rows and strictly increasing down the columns. Note that our standard tableaux are called semistandard, for example in [12]. Let $\chi_{i}(T)$ denote the number of entries equal to $i$ that appear in $T$. Then the tuple $\chi(T)=$ $\left(\chi_{1}(T), \chi_{2}(T), \ldots, \chi_{n}(T)\right)$ is called the content of $T$. The row sequence of $T$ is obtained by reading off the entries of $T$ across the rows, working from left to right and top to bottom; this sequence shall be denoted $I_{R}(T)$. The column sequence of $T$ is obtained by reading off the entries of $T$ down the columns, again from top to bottom and from left to right; we denote this sequence by $I_{C}(T)$.

Let $T(\lambda)$ denote the $\lambda$-tableau in which the $i$-th row consists entirely of $i$ s and let $I_{R}(T(\lambda))=I(\lambda)$ and $I_{C}(T(\lambda))=I_{C}(\lambda)$. We will often write $T=T_{I}$ where $I=I_{C}(T)$.

Let $I(n, r)=\left\{I=\left(i_{1}, i_{2}, \ldots, i_{r}\right) \mid i_{j} \in\{1, \ldots, n\}, 1 \leq j \leq r\right\}$. The symmetric group $S_{r}$ acts on $I(n, r)$ as place permutations;

$$
I \sigma=\left(i_{1 \sigma}, i_{2 \sigma}, \ldots, i_{r \sigma}\right), I=\left(i_{1}, i_{2}, \ldots, i_{r}\right) \in I(n, r), \sigma \in S_{r} .
$$

This gives an action on the set of $\lambda$-tableaux by $T_{I} \sigma=T_{I \sigma}$. Define the $\lambda$-tableau $T^{\lambda}$ by $I_{R}\left(T^{\lambda}\right)=(1,2, \ldots, r)$. Let $C(\lambda)$ be the subgroup of $S_{r}$ given by the permutations that leave the columns of $T^{\lambda}$ invariant. Similarly, $R(\lambda)$ is the subgroup of $S_{r}$ that consists of all permutations that leave the columns of $T^{\lambda}$ invariant.

Example. Let $\lambda=(3,2)$. Then $I(\lambda)=(1,1,1,2,2)$ and $I_{C}(\lambda)=(1,2,1,2,1)$;

$$
T^{\lambda}=\begin{array}{|l|l|l|}
\hline 1 & 2 & 3 \\
\hline 4 & 5 &
\end{array}
$$

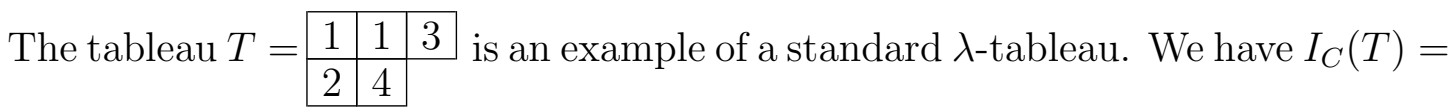
$(1,2,1,4,3)$ and $I_{R}(T)=(1,1,3,2,4)$. When we write $T=T_{I}$ for this $T$, we mean $I=(1,2,1,4,3)$. 


\section{The $q$-Schur algebra And quantum Codeterminants}

Let $K$ be a field, and let $q$ be a non-zero element of $K$. Following Parshall-Wang [14], Taft-Towber [17], Manin [15], the coordinate ring of quantum matrices, which we denote by $A_{q}(n)$, is defined to be the $K$-algebra generated by $n^{2}$ variables $x_{i j}$, $1 \leq i, j \leq n$, subject to the relations

$$
\begin{array}{ll}
x_{i l} x_{i k}=q x_{i k} x_{i l} & 1 \leq k<l \leq n \\
x_{j k} x_{i k}=q x_{i k} x_{j k} & 1 \leq i<j \leq n \\
x_{i l} x_{j k}=x_{j k} x_{i l} & 1 \leq i<j \leq n, 1 \leq k<l \leq n \\
x_{i k} x_{j l}-x_{j l} x_{i k}=\left(q^{-1}-q\right) x_{i l} x_{j k} & 1 \leq i<j \leq n, 1 \leq k<l \leq n .
\end{array}
$$

The algebra $A_{q}(n)$ is a co-algebra, with comultiplication given by

$$
\Delta\left(x_{i j}\right)=\sum_{k=1}^{n} x_{i k} \otimes x_{k j} .
$$

Given $I=\left(i_{1}, i_{2}, \ldots, i_{r}\right), J=\left(j_{1}, j_{2}, \ldots, j_{r}\right) \in I(n, r)$, define

$$
x_{I, J}=x_{i_{1}, j_{1}} \cdots x_{i_{r}, j_{r}} .
$$

Let $A_{q}(n, r)$ be the $K$-subspace of $A_{q}(n)$ generated by monomials $x_{I, J}, I, J \in$ $I(n, r)$. Then $A_{q}(n, r)$ is a subcoalgebra of $A_{q}(n)$, and we define the $q$-Schur algebra $S_{q}(n, r)$ to be the dual $A_{q}(n, r)^{*}$. Then $S_{q}(n, r)$ is an associative algebra.

Let $V$ be the $K$-vector space $K^{n}$. For historical purposes, we will give the connection between the Parshall-Wang and the Dipper-James versions of the $q$ Schur algebra. Following Jimbo (see also [14] 11.3) $V^{\otimes r}$ is a module for the Hecke algebra $\mathcal{H}_{q}(r)$, and it is shown in [14], Theorem 11.3.1, that

$$
S_{q}(n, r) \cong \operatorname{End}_{\mathcal{H}_{q}(r)}\left(V^{\otimes r}\right) \text {. }
$$

It then follows as in $\mathrm{Du}[6]$ that $S_{q}(n, r)$ is isomorphic to the $q^{-2}$-Schur algebra as defined by Dipper and James [3].

Let

$\mathcal{I}^{2}(n, r)=\left\{(I, J) \in I(n, r) \times I(n, r) \mid i_{1} \leq i_{2} \leq \cdots \leq i_{r}\right.$ and $j_{k} \leq j_{k+1}$ if $\left.i_{k}=i_{k+1}\right\}$.

The set $\mathcal{B}=\left\{x_{I, J} \mid(I, J) \in \mathcal{I}^{2}(n, r)\right\}$ forms a basis for $A_{q}(n, r)$ (see, for instance [4, section 1.1] although the relations used there differ from ours.) The Schur algebra $S_{q}(n, r)$ has the basis $\left\{\xi_{I, J} \mid(I, J) \in \mathcal{I}^{2}(n, r)\right\}$ dual to the basis $\mathcal{B}$ of $A_{q}(n, r)$. Thus, if $(A, B),(I, J) \in \mathcal{I}^{2}(n, r)$, then $\xi_{I, J}\left(x_{A, B}\right)=1$ if $x_{A, B}=x_{I, J}$ and $\xi_{I, J}\left(x_{A, B}\right)=0$ otherwise. For arbitrary $(P, Q) \in I(n, r) \times I(n, r)$, define

$$
\xi_{P, Q}=\sum_{(I, J) \in \mathcal{I}^{2}(n, r)} \alpha_{I, J} \xi_{I, J}
$$

where

$$
x_{P, Q}=\sum_{(I, J) \in \mathcal{I}^{2}(n, r)} \alpha_{I, J} x_{I, J} .
$$

Note that for $(I, J),(A, B) \in I(n, r) \times I(n, r)$, we have

$$
\xi_{I, J}\left(x_{A, B}\right)=0 \text { unless } I=A \sigma \text { and } J=B \tau \text { for } \sigma, \tau \in S_{r},
$$


but, unlike in the classical case, it is not necessary that $\sigma=\tau$.

For elements $I$ and $J$ of $I(n, r)$, we define $I<J$ if $I$ is less than $J$ in the lexicographic order. For pairs $(A, B),(I, J)$ where $A, B, I, J \in I(n, r)$, we define $(A, B)<(I, J)$ if $A<I$ or $A=I$ and $B<J$.

The symmetric group acts on the set of pairs $(I, J)$, where $I, J \in I(n, r)$, by $(I, J) \sigma=(I \sigma, J \sigma)$. For any such pair $(I, J)$, the minimal element in the $S_{r}$-orbit containing $(I, J)$ is denoted $(I, J)_{0}$; this $(I, J)_{0}$ is in $\mathcal{I}^{2}(n, r)$.

The quantum determinant $D$ of the $n \times n$ matrix $X=\left(x_{i j}\right)$ is defined by

$$
D=\sum_{\sigma \in S_{n}}(-q)^{-l(\sigma)} x_{\sigma(1) 1} x_{\sigma(2) 2} \cdots x_{\sigma(n) n}
$$

where $l$ is the standard length function on the symmetric group $S_{n}$.

Let $I, J \in I(n, r)$ where $I$ is increasing. Define the quantum minor $D(I, J)$ by

$$
D(I, J)=\sum_{\sigma \in S_{r}}(-q)^{-\ell(\sigma)} x_{I \sigma, J}
$$

Let $S$ and $T$ be $\lambda$-tableaux with $S$ column increasing. Let $S(i)$ be the sequence given by the entries in the $i$-th column of $S$, with similar notation $T(i)$ for $T$. The quantum bideterminant $X_{I, J}^{\lambda}$, where $I=I_{C}(S), J=I_{C}(T)$, is defined to be the product

$$
X_{I, J}^{\lambda}=D(S(1), T(1)) D(S(2), T(2)) \cdots D(S(k), T(k))=\sum_{\sigma \in C(\lambda)}(-q)^{-\ell(\sigma)} x_{I \sigma, J}
$$

where $k$ is the number of columns of $S$ (and of $T$ ). From [17, Proposition 2.1], we have $D(I, J)=0$ if $J$ has repeated entries, so if $T=T_{J}$ has repeated entries in a column then $X_{I, J}^{\lambda}=0$. Also in [17, Proposition 2.1], it is proved that $D(I, J \tau)=$ $(-q)^{-\ell(\tau)} D(I, J)$, for $\tau \in S_{r}$, from which it follows that

$$
X_{I, J \tau}^{\lambda}=(-q)^{-\ell(\tau)} X_{I, J} \text { for } \tau \in C(\lambda) .
$$

Define $\Lambda^{+}(n, r)$ to be the set of partitions of $r$ into at most $n$ parts. Let $\mathcal{I}_{\lambda}=$ $\left\{I \in I(n, r) \mid T_{I}\right.$ is standard $\}$ and let

$$
\mathcal{S}=\left\{(\lambda, I, J) \mid \lambda \in \Lambda^{+}(n, r), I, J \in \mathcal{I}_{\lambda}\right\} .
$$

The following theorem is due to Huang, Zhang [11].

Theorem 1. The set $\left\{X_{I, J}^{\lambda} \mid(\lambda, I, J) \in \mathcal{S}\right\}$ forms a basis for $A_{q}(n, r)$.

Since $S_{q}(n, r)$ is the dual of $A_{q}(n, r)$, we have a natural pairing

$$
\langle,\rangle: A_{q}(n, r) \times S_{q}(n, r) \rightarrow K
$$

where $\left\langle x_{A, B}, \xi_{I, J}\right\rangle=\xi_{I, J}\left(x_{A, B}\right)$. We also have the transpose operator $\operatorname{tr}: A_{q}(n) \rightarrow$ $A_{q}(n)$ defined by $\operatorname{tr}\left(x_{I, J}\right)=x_{J, I}$. This is a $K$-linear map since it preserves the relations (1). It yields a transpose operator on $S_{q}(n, r)$ defined by $\left\langle P, \operatorname{tr}\left(\xi_{I, J}\right)\right\rangle=$ $\left\langle\operatorname{tr}(P), \xi_{I, J}\right\rangle$.

For the next result, if $I$ and $J$ are in $I(n, r)$, we let $x_{(I, J)}$ denote $x_{I, J}$.

Lemma 2. Suppose that $(A, B) \in \mathcal{I}^{2}(n, r)$. Then $x_{B, A}=x_{(B, A)_{0}}$. 
Proof. This is certainly true if $(B, A) \in \mathcal{I}^{2}(n, r)$, so suppose that $(B, A) \notin \mathcal{I}^{2}(n, r)$. One possibility is that the entries of $B$ are not increasing. Suppose that the $i$-th entry $b_{i}$ of $B$ is greater than $b_{i+1}$. We cannot have $a_{i}=a_{i+1}$, since if we did, then $(A, B) \notin \mathcal{I}^{2}(n, r)$. Since the entries of $A$ are weakly increasing, then $a_{i}<a_{i+1}$. Then the third equation of (1) gives

$$
x_{b_{i}, a_{i}} x_{b_{i+1}, a_{i+1}}=x_{b_{i+1}, a_{i+1}} x_{b_{i}, a_{i}} \text { and } x_{a_{i}, b_{i}} x_{a_{i+1}, b_{i+1}}=x_{a_{i+1}, b_{i+1}} x_{a_{i}, b_{i}} .
$$

Let $B^{\prime}$ and $A^{\prime}$ be the sequences obtained from $B$ and $A$ (respectively) by switching the entries in positions $i$ and $i+1$. So we have $x_{B^{\prime}, A^{\prime}}=x_{B, A}$. Then $\left(B^{\prime}, A^{\prime}\right)<$ $(B, A)$, and $x_{A^{\prime}, B^{\prime}}=x_{A, B}$ so by induction, we have

$$
x_{B^{\prime}, A^{\prime}}=x_{(B, A)_{0}} .
$$

It follows that $x_{B, A}=x_{(B, A)_{0}}$.

A second possibility, if $(B, A) \notin \mathcal{I}^{2}(n, r)$, is that for some $i, b_{i}=b_{i+1}$ and $a_{i}>a_{i+1}$. This is not possible, since the entries of $A$ are weakly increasing. This proves the lemma.

Lemma 3. For $A, B \in I(n, r),(I, J) \in \mathcal{I}^{2}(n, r)$, we have

$$
\left\langle x_{A, B}, \xi_{I, J}\right\rangle=\left\langle x_{B, A}, \xi_{J, I}\right\rangle \text {. }
$$

Proof. It follows from the previous lemma that if $(I, J) \in \mathcal{I}^{2}(n, r)$, then $\xi_{J, I}=$ $\xi_{(J, I)_{0}}$. Write

$$
x_{A, B}=\sum_{(P, Q) \in \mathcal{I}^{2}(n, r)} c_{P, Q} x_{P, Q} .
$$

Since the transpose map is linear, the previous lemma implies that

$$
\begin{aligned}
\left\langle x_{B, A}, \xi_{J, I}\right\rangle & =\left\langle\sum_{(P, Q) \in \mathcal{I}^{2}(n, r)} c_{P, Q} x_{(Q, P)_{0}}, \xi_{(J, I)_{0}}\right\rangle \\
& =c_{I, J}=\left\langle x_{A, B}, \xi_{I, J}\right\rangle .
\end{aligned}
$$

For $\lambda$-tableaux $S$ and $T$ we let $A=I_{R}(S)$ and $B=I_{R}(T)$ and define the quantum codeterminant $Y_{A, B}^{\lambda}$ by

$$
Y_{A, B}^{\lambda}=\xi_{A, I(\lambda)} \xi_{I(\lambda), B}
$$

Note that, when associating a quantum bideterminant to a pair of tableaux we read down the columns while when associating a quantum codeterminant to a pair of tableaux we read across the rows. This ensures that a given codeterminant is a product of a basis element in $S_{q}(n, r)$ and the transpose of a basis element in $S_{q}(n, r)$.

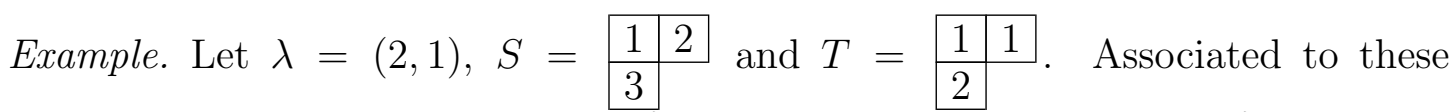
tableaux we have a bideterminant $X_{(1,3,2),(1,2,1)}^{\lambda}$ and a codeterminant $Y_{(1,2,3),(1,1,2)}^{\lambda}=$ $\xi_{(1,2,3),(1,1,2)} \xi_{(1,1,2),(1,1,2)}$.

We will require the following proposition in Section 5. 
Proposition 4. Let $S$ be a column increasing $\lambda$-tableau with $I=I_{C}(S)$ and let $J \in I(n, r)$. Then if $A, B \in I(n, r)$ we have

$$
\left\langle X_{I, J}^{\lambda}, Y_{A, B}^{\lambda}\right\rangle=\left\langle X_{I, I_{C}(\lambda)}^{\lambda}, \xi_{A, I(\lambda)}\right\rangle\left\langle X_{I_{C}(\lambda), J}^{\lambda}, \xi_{I(\lambda), B}\right\rangle .
$$

Proof. Multiplication in the $q$-Schur algebra is defined using the comultiplication $\Delta$ in $A_{q}(n, r)$, so

$$
\begin{aligned}
\left\langle X_{I, J}^{\lambda}, Y_{A, B}^{\lambda}\right\rangle & =\left\langle\sum_{\sigma \in C(\lambda)}(-q)^{-\ell(\sigma)} x_{I \sigma, J}, Y_{A, B}^{\lambda}\right\rangle \\
& =\sum_{\sigma \in C(\lambda)}(-q)^{-\ell(\sigma)}\left\langle x_{I \sigma, J}, \xi_{A, I(\lambda)} \xi_{I(\lambda), B}\right\rangle \\
& =\sum_{\sigma \in C(\lambda)} \sum_{S \in I(n, r)}(-q)^{-\ell(\sigma)}\left\langle x_{I \sigma, S}, \xi_{A, I(\lambda)}\right\rangle\left\langle x_{S, J}, \xi_{I(\lambda), B}\right\rangle \\
& =\sum_{S \in I(n, r)}\left\langle\sum_{\sigma \in C(\lambda)}(-q)^{-\ell(\sigma)} x_{I \sigma, S}, \xi_{A, I(\lambda)}\right\rangle\left\langle x_{S, J}, \xi_{I(\lambda), B}\right\rangle
\end{aligned}
$$

Denote the double sum on the previous line by $\mathcal{S}$. Suppose that $\left\langle x_{S, J}, \xi_{I(\lambda), B}\right\rangle \neq 0$. Then $S=I(\lambda) \alpha$ for some permutation $\alpha \in S_{r}$. Since $I(\lambda)=I_{C}(\lambda) \beta$ for some $\beta \in S_{r}$, we have $S=I_{C}(\lambda) \tau$ for some $\tau \in S_{r}$.

We shall show that if $\tau \notin C(\lambda) R(\lambda)$ then

$$
\sum_{\sigma \in C(\lambda)}(-q)^{-\ell(\sigma)} x_{I \sigma, I_{C}(\lambda) \tau}=0 .
$$

If $\tau \notin C(\lambda) R(\lambda)$ then, since all entries in each row of $T(\lambda)$ are equal, $\tau$ must move some element of $T(\lambda)$ to a different column. Consider the left-most column, say column $i$, of $T(\lambda)$ which is not kept invariant by $\tau$. If the length of this column is $j$, then the elements of this column are $1,2, \ldots, j$. Since the first $i-1$ columns are invariant under $\tau$, and since the columns to the right of the $i$-th column are not longer than the $i$-th column, then after applying $\tau$ there must be a repeated entry in column $i$ of $T(\lambda) \tau$, and so

$$
\sum_{\sigma \in C(\lambda)}(-q)^{-\ell(\sigma)} x_{I \sigma, I_{C}(\lambda) \tau}=0
$$

as claimed.

Hence, in the double sum $\mathcal{S}$, we need only sum over those $S$ in $I(n, r)$ such that $S=I_{C}(\lambda) \tau$ with $\tau \in C(\lambda) R(\lambda)$. Since the elements in each row of $T(\lambda)$ are all the same, $R(\lambda)$ has no effect on $I_{C}(\lambda)$, and since the elements of each column of $T(\lambda)$ are all different, distinct elements $\tau \in C(\lambda)$ give distinct $S=I_{C}(\lambda) \tau$. Then

$$
\mathcal{S}=\sum_{\tau \in C(\lambda)} \sum_{\sigma \in C(\lambda)}(-q)^{-\ell(\sigma)}\left\langle x_{I \sigma, I_{C}(\lambda) \tau}, \xi_{A, I(\lambda)}\right\rangle\left\langle x_{I_{C}(\lambda) \tau, J}, \xi_{I(\lambda), B}\right\rangle
$$

By (2),

$$
\sum_{\sigma \in C(\lambda)}(-q)^{-\ell(\sigma)} x_{I \sigma, I_{C}(\lambda) \tau}=(-q)^{-\ell(\tau)} \sum_{\sigma \in C(\lambda)}(-q)^{-\ell(\sigma)} x_{I \sigma, I_{C}(\lambda)}, \text { for } \tau \in C(\lambda) .
$$


Therefore

$$
\mathcal{S}=\sum_{\sigma \in C(\lambda)} \sum_{\tau \in C(\lambda)}(-q)^{-\ell(\sigma)-\ell(\tau)}\left\langle x_{I \sigma, I_{C}(\lambda)}, \xi_{A, I(\lambda)}\right\rangle\left\langle x_{I_{C}(\lambda) \tau, J}, \xi_{I(\lambda), B}\right\rangle .
$$

This is equal to

$$
\left\langle X_{I, I_{C}(\lambda)}^{\lambda}, \xi_{A, I(\lambda)}\right\rangle\left\langle X_{I_{C}(\lambda), J}^{\lambda}, \xi_{I(\lambda), B}\right\rangle
$$

\section{The QUantum hyperalgebra And the QUANtum DéSARMÉnien MATRIX}

In this section we recall the definition of the quantum Désarménien matrix from [16] as well as some relevant results from [12].

Let $q$ be an indeterminate, $\mathcal{A}=\mathbb{Z}\left[q, q^{-1}\right]$ the ring of Laurent polynomials in $q$, and $\mathbb{Q}(q)$ the field of quotients of $\mathcal{A}$. Let $U_{\mathbb{Q}(q)}$ denote the quantum enveloping algebra of $g l(n)$, which has generators $E_{i}, F_{i}, K_{j}, K_{j}^{-1}$ with $1 \leq i<n, 1 \leq j \leq n$ and relations given, for example, in [12, Section 3.]

Define $K_{i, i+1}=K_{i} K_{i+1}^{-1}$. We have a comultiplication $\Delta: U_{\mathbb{Q}(q)} \rightarrow U_{\mathbb{Q}(q)} \otimes U_{\mathbb{Q}(q)}$ defined by

(3) $\Delta\left(E_{i}\right)=1 \otimes E_{i}+E_{i} \otimes K_{i, i+1}, \quad \Delta\left(F_{i}\right)=K_{i, i+1}^{-1} \otimes F_{i}+F_{i} \otimes 1, \quad \Delta\left(K_{i}\right)=K_{i} \otimes K_{i}$.

We then have an algebra homomorphism $\Delta^{r-1}: U_{\mathbb{Q}(q)} \rightarrow U_{\mathbb{Q}(q)}^{\otimes r}$ that satisfies

$\Delta^{r-1}\left(E_{i}\right)=1 \otimes \cdots \otimes 1 \otimes E_{i}+1 \otimes \cdots \otimes 1 \otimes E_{i} \otimes K_{i, i+1}+\cdots+E_{i} \otimes K_{i, i+1} \otimes \cdots \otimes K_{i, i+1}$, $\Delta^{r-1}\left(F_{i}\right)=F_{i} \otimes 1 \otimes \cdots \otimes 1+K_{i, i+1}^{-1} \otimes F_{i} \otimes \cdots \otimes 1+\cdots+\otimes K_{i, i+1}^{-1} \otimes \cdots \otimes K_{i, i+1}^{-1} \otimes F_{i}$.

To define Lusztig's integral form, let $a$ be a non-negative integer and define

$$
[a] !=\prod_{k=1}^{a} \frac{q^{k}-q^{-k}}{q-q^{-1}} \text { and }\left(\begin{array}{c}
K_{j} \\
a
\end{array}\right)=\prod_{s=1}^{a} \frac{q^{-s+1} K_{j}-q^{s-1} K_{j}^{-1}}{q^{s}-q^{-s}} .
$$

For $X \in U_{\mathbb{Q}(q)}$ and $a \in \mathbb{N}$, we have the divided power $X^{(a)}=\frac{X^{a}}{[a] !}$. Let $U_{\mathcal{A}}$ denote the Hopf $\mathcal{A}$-subalgebra of $U_{\mathbb{Q}(q)}$ generated by the the elements

$$
E_{i}^{(a)}, F_{i}^{(a)}, K_{j}, K_{j}^{-1},\left(\begin{array}{c}
K_{j} \\
a
\end{array}\right), a \in \mathbb{N}, 1 \leq i<n, 1 \leq j \leq n .
$$

The quantum hyperalgebra $U_{K}$ is defined by base change; $U_{K}=U_{\mathcal{A}} \otimes_{\mathcal{A}} K$. Note that $K$ is an $\mathcal{A}$-algebra by defining $q \in \mathcal{A}$ to act on $K$ by multiplication by some unit $t \in K$. When we write $u \in U_{K}$, we are referring to the image of $u$ in $U_{K}$ via the map $\phi: U_{\mathcal{A}} \rightarrow U_{K}$ defined by $\phi(u)=u \otimes 1$.

Let $A_{\mathcal{A}}(n)$ be the $\mathcal{A}$-algebra generated by the variables $x_{i j}, 1 \leq i, j \leq n$ subject to the relations (1). Then $A_{q}(n)=A_{\mathcal{A}}(n) \otimes_{\mathcal{A}} K$. The following action makes $A_{\mathcal{A}}(n)$ a $U_{\mathcal{A}}$-module:

$$
E_{i} x_{k l}=\delta_{i+1, l} x_{k i}, \quad F_{i} x_{k l}=\delta_{i l} x_{k, i+1}, \quad K_{i} x_{k l}=q^{\delta_{i l}} x_{k l}, \quad K_{i}^{-1} x_{k l}=q^{-\delta_{i l}} x_{k l}
$$

and $E_{i}(P Q)=P\left(E_{i} Q\right)+\left(E_{i} P\right)\left(K_{i, i+1} Q\right), F_{i}(P Q)=\left(K_{i, i+1}^{-1} P\right)\left(F_{i} Q\right)+\left(F_{i} P\right) Q$, $K_{i}(P Q)=\left(K_{i} P\right)\left(K_{i} Q\right)$ where $P, Q \in A_{\mathcal{A}}(n)$. 
Furthermore, for a fixed $\lambda$, the $\mathcal{A}$-span of the quantum bideterminants of the

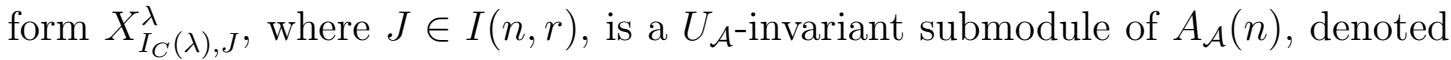
by $\nabla_{\mathcal{A}}(\lambda)$. The $A_{q}(n)$-module obtained by base change, denoted $\nabla_{q}(\lambda)$, is called the $q$-Schur module. Note that in [16] and [12], $X_{I_{C}(\lambda), J}^{\lambda}$ is denoted by $\left[T_{J}\right]$. From [10], for instance, we have the following.

Theorem 5. The set $\left\{X_{I_{C}(\lambda), J}^{\lambda} \mid J \in \mathcal{I}_{\lambda}\right\}$ forms a $K$-basis for $\nabla_{q}(\lambda)$.

Let $|i-j| \geq 1$ and define $E_{i j}, F_{i j} \in U_{K}$ recursively as follows:

$E_{i}=E_{i, i+1}, E_{i j}=E_{i} E_{i+1, j}-q^{-1} E_{i+1, j} E_{i}, F_{i}=F_{i, i+1}, F_{i j}=F_{i+1, j} F_{i}-q^{-1} F_{i} F_{i+1, j}$.

From [13, Proposition 41.1.3], it follows that if $a$ is a positive integer, then $E_{i j}^{(a)}$ and $F_{i j}^{(a)}$ belong to $U_{\mathcal{A}}$, and hence by base change, to $U_{K}$.

To a standard $\lambda$-tableau $T$ with $k \leq n$ rows, define elements $E_{T}, F_{T} \in U_{K}$ by

$$
\begin{aligned}
& E_{T}=\prod_{1 \leq i<k, i<j \leq n} E_{i j}^{\left(\gamma_{i j}\right)}=E_{k-1, k}^{\left(\gamma_{k-1, k}\right)} \cdots E_{2 k}^{\left(\gamma_{2 k}\right)} \cdots E_{23}^{\left(\gamma_{23}\right)} E_{1 k}^{\left(\gamma_{1 k}\right)} \cdots E_{13}^{\left(\gamma_{13}\right)} E_{12}^{\left(\gamma_{12}\right)}, \\
& F_{T}=\prod_{1 \leq i<k, i<j \leq n} F_{i j}^{\left(\gamma_{i j}\right)}=F_{12}^{\left(\gamma_{12}\right)} F_{13}^{\left(\gamma_{13}\right)} \cdots F_{1 k}^{\left(\gamma_{1 k}\right)} F_{23}^{\left(\gamma_{23}\right)} \cdots F_{2 k}^{\left(\gamma_{2 k}\right)} \cdots F_{k-1, k}^{\left(\gamma_{k-1, k}\right)},
\end{aligned}
$$

where $\gamma_{i j}$ is the number of entries equal to $j$ in row $i$ of $T$, and $k$ is the number of columns in $T$.

Definition 6. Given a standard $\lambda$-tableau $T$ and a column increasing $\lambda$-tableau $S$, with $J=I_{C}(S)$, define $\Omega_{q}(S, T)=c$ where $c$ is the coefficient of $X_{I_{C}(\lambda), I_{C}(\lambda)}^{\lambda}$ in the expansion of $E_{T} X_{I_{C}(\lambda), J}^{\lambda}$ into a linear combination of the basis elements in Theorem 5 .

Given $\lambda$-tableaux $T_{I}$ and $T_{J}$, we say $T_{I}<T_{J}$ if $I<J$ with respect to the lexicographic order on $I(n, r)$.

Definition 7. The quantum Désarménien matrix is the matrix

$$
\Omega_{q}=\left[\Omega_{q}\left(T_{I}, T_{J}\right)\right]_{I, J \in \mathcal{I}_{\lambda}} .
$$

We have the following theorem from [16, Theorem 4.10].

Theorem 8. Let $T_{I}$ and $T_{J}$ be column increasing $\lambda$-tableaux with $T_{J}$ standard. If $\Omega_{q}\left(T_{I}, T_{J}\right) \neq 0$ then $I \leq J$.

For a $\lambda$-tableau $T$, let $t_{i j}$ denote the entry in the $i$-th row and $j$-th column of T. Define

$$
s(T)=\left|\left\{\left(\left(i, t_{i a}\right),\left(j, t_{j b}\right)\right) \mid i>j, a<b, t_{i a}=t_{j b}\right\}\right| .
$$

Thus, $s(T)$ is the number of pairs $(i a, j b)$ where $t_{i a}=t_{j b}$ and $t_{i a}$ sits in a row below $t_{j b}$ and in a column to the left of $t_{j b}$.

Example. If $T=$\begin{tabular}{|l|l|l|}
\hline 2 & 5 & 5 \\
\hline 5 & 6 & , then \\
\end{tabular}

The following theorem, [12, Theorem 5.12], states that the entries of the quantum Désarménien matrix can be found using the $q$-Schur algebra. 
Theorem 9. Let $T_{I}$ and $T_{J}$ be column increasing $\lambda$-tableaux with $T_{J}$ standard. If $T_{I}$ and $T_{J}$ have the same content then

$$
\Omega_{q}\left(T_{I}, T_{J}\right)=q^{-s\left(T_{J}\right)}\left\langle X_{I_{C}(\lambda), I}^{\lambda}, \xi_{I(\lambda), I_{R}\left(T_{J}\right)}\right\rangle .
$$

In the proof of Theorem 12 we will also use the following Lemma [12, Lemma $5.8]$.

Lemma 10. Let $T_{J}$ be a standard $\lambda$-tableau. Then

$$
\left\langle X_{I_{C}(\lambda), J}^{\lambda}, \xi_{I(\lambda), I_{R}\left(T_{J}\right)}\right\rangle=q^{s\left(T_{J}\right)} .
$$

Example. Let $\lambda=(3,2)$ and $T=$\begin{tabular}{|l|l|l|}
\hline 2 & 5 & 5 \\
\hline 5 & 6 &
\end{tabular} as above. Then

$$
\left\langle X_{(1,2,1,2,1),(2,5,5,6,5)}^{\lambda}, \xi_{(1,1,1,2,2),(2,5,5,5,6)}\right\rangle=q^{2} .
$$

\section{A BASIS OF CODETERMINANTS FOR $S_{q}(n, r)$}

Partially order $\Lambda^{+}(n, r)$ as follows:

$$
\lambda \unlhd \mu \text { if } \lambda_{1}+\lambda_{2}+\cdots+\lambda_{p} \leq \mu_{1}+\mu_{2}+\cdots+\mu_{p} \text { for all } p \in\{1,2, \ldots, n\} .
$$

Here, if $\lambda$ is a partition of $r$ into $k<n$ parts, we let $\lambda_{p}=0$ for $p>k$ (similarly for $\mu)$. Recall that $\mathcal{S}=\left\{(\lambda, I, J) \mid \lambda \in \Lambda^{+}(n, r)\right.$ and $T_{I}, T_{J}$ are standard $\}$.

Definition 11. For $(\lambda, I, J),(\mu, A, B) \in \mathcal{S}$, define $(\lambda, I, J)<(\mu, A, B)$ if $\lambda \unlhd \mu$ or $\lambda=\mu$ and $(I>A$ or $I=A$ and $J>B)$.

Keep in mind that the codeterminants come from reading across rows of standard tableaux. In the following definition, for $A \in I(n, r)$, let $A^{\prime}=I_{R}\left(T_{A}\right)$. In other words, if $A=I_{C}(T)$, we let $A^{\prime}=I_{R}(T)$.

Define a matrix $\mathcal{D}_{q}$ as

$$
\mathcal{D}_{q}=\left(\left\langle X_{I, J}^{\lambda}, Y_{A^{\prime}, B^{\prime}}^{\mu}\right\rangle\right)_{(\lambda, I, J),(\mu, A, B) \in \mathcal{S}} .
$$

Theorem 12. The matrix $\mathcal{D}_{q}$ is upper triangular, using the order in Definition 11, with nonzero entries on the diagonal.

Proof. We first consider the diagonal entries of $\mathcal{D}_{q}$. Let $(\lambda, I, J) \in \mathcal{S}$. Using Proposition 4, we have $\left\langle X_{I, J}^{\lambda}, Y_{I^{\prime}, J^{\prime}}^{\lambda}\right\rangle=\left\langle X_{I, I_{C}(\lambda)}^{\lambda}, \xi_{I^{\prime}, I(\lambda)}\right\rangle\left\langle X_{I_{C}(\lambda), J}^{\lambda}, \xi_{I(\lambda), J^{\prime}}\right\rangle$. But by Lemmas 3 and 10, $\left\langle X_{I, I_{C}(\lambda)}^{\lambda}, \xi_{I^{\prime}, I(\lambda)}\right\rangle=\left\langle X_{I_{C}(\lambda), I}^{\lambda}, \xi_{I(\lambda), I^{\prime}}\right\rangle=q^{s\left(T_{I}\right)}$ and $\left\langle X_{I_{C}(\lambda), J}, \xi_{I(\lambda), J^{\prime}}\right\rangle=$ $q^{s\left(T_{J}\right)}$. Thus $\left\langle X_{I, J}^{\lambda}, Y_{I^{\prime}, J^{\prime}}^{\lambda}\right\rangle=q^{s\left(T_{I}\right)+s\left(T_{J}\right)} \neq 0$.

Now suppose that $(\lambda, I, J),(\mu, A, B) \in \mathcal{S}$ with $(\lambda, I, J)<(\mu, A, B)$. Suppose first that $\lambda=\mu$. Using Proposition 1 and Theorem 9,

$$
\begin{aligned}
\left\langle X_{I, J}^{\lambda}, Y_{A^{\prime}, B^{\prime}}^{\lambda}\right\rangle & =\left\langle X_{I, I_{C}(\lambda)}^{\lambda}, \xi_{A^{\prime}, I(\lambda)}\right\rangle\left\langle X_{I_{C}(\lambda), J^{\prime}}^{\lambda}, \xi_{I(\lambda), B^{\prime}}\right\rangle \\
& =q^{s\left(T_{A}\right)} \Omega_{q}\left(T_{I}, T_{A}\right) q^{s\left(T_{B}\right)} \Omega_{q}\left(T_{J}, T_{B}\right) .
\end{aligned}
$$

But, since $(\lambda, I, J)<(\lambda, A, B)$, either $I>A$ or $I=A$ and $J>B$. In the former case, $\Omega_{q}\left(T_{I}, T_{A}\right)=0$ by Theorem 8 , and in the latter case, $\Omega_{q}\left(T_{J}, T_{B}\right)=0$. Thus $\left\langle X_{I, J}^{\lambda}, Y_{A^{\prime}, B^{\prime}}^{\lambda}\right\rangle=0$. 
Suppose that $\lambda \triangleleft \mu$ and let $p$ be the smallest positive integer with $\mu_{1}+\mu_{2}+$ $\cdots+\mu_{p}>\lambda_{1}+\lambda_{2}+\cdots+\lambda_{p}$. As in the proof of Proposition 4, we have

$$
\left\langle X_{I, J}^{\lambda}, Y_{A^{\prime}, B^{\prime}}^{\mu}\right\rangle=\sum_{\sigma \in C(\lambda)} \sum_{S \in I(n, r)}(-q)^{-\ell(\sigma)}\left\langle x_{I \sigma, S}, \xi_{A^{\prime}, I(\mu)}\right\rangle\left\langle x_{S, J}, \xi_{I(\mu), B^{\prime}}\right\rangle
$$

which is equal to $\sum_{S \in I(n, r)}\left\langle X_{I, S}^{\lambda}, \xi_{A^{\prime}, I(\mu)}\right\rangle\left\langle x_{S, J}, \xi_{I(\mu), B^{\prime}}\right\rangle$. If $\left\langle X_{I, J}^{\lambda}, Y_{A^{\prime}, B^{\prime}}^{\mu}\right\rangle \neq 0$ then, for some $S \in I(n, r)$, we have $\left\langle X_{I, S}^{\lambda}, \xi_{A^{\prime}, I(\mu)}\right\rangle \neq 0$. Since

$$
X_{I, S}^{\lambda}=\sum_{\sigma \in C(\lambda)}(-q)^{-\ell(\sigma)} x_{I \sigma, S},
$$

then $I(\mu)=S \alpha$ where $\alpha \in S_{r}$. Thus $S$ contains $\mu_{i}$ entries equal to $i$ for $1 \leq i \leq n$. We have $\mu_{i}=\lambda_{i}$ for $1 \leq i \leq p-1$, but $\mu_{p}>\lambda_{p}$. Thus $T_{S}$ contains an $i$ in each of the $\lambda_{i}$ columns for $1 \leq i \leq p-1$ and since $\mu_{p}>\lambda_{p}$, this forces at least two entries equal to $p$ in the same column of $T_{S}$. It follows that $X_{I, S}^{\lambda}=0$ and so $\left\langle X_{I, S}^{\lambda}, \xi_{A^{\prime}, I(\mu)}\right\rangle=0$ and, consequently, $\left\langle X_{I, J}^{\lambda}, Y_{A^{\prime}, B^{\prime}}^{\mu}\right\rangle=0$.

Note that, unlike in the classical case, the entries on the diagonal of the matrix $\mathcal{D}_{q}$ may not necessarily be equal to one; they are, in fact, of the form $q^{k}, k \geq 0$. The following Corollary follows easily from Theorem 12, as in [7].

Corollary 13. The set $\left\{Y_{A, B}^{\mu} \mid(\mu, A, B) \in \mathcal{S}\right\}$ forms a basis for $S_{q}(n, r)$.

Proof. Let $\mathcal{B}=\left\{X_{I, J}^{\lambda} \mid(\lambda, I, J) \in \mathcal{S}\right\}$ be the basis for $A_{q}(n, r)$ listed in Theorem 1 and let $\mathcal{B}^{\prime}=\left\{g_{I, J}^{\lambda} \mid(\lambda, I, J)\right\}$ be the basis of $S_{q}(n, r)$ that is dual to $\mathcal{B}$. Since $\mathcal{D}_{q}$ is an invertible matrix and $Y_{A, B}^{\mu}=\sum_{(\lambda, I, J) \in \mathcal{S}}\left\langle X_{I, J}^{\lambda}, Y_{A, B}^{\mu}\right\rangle g_{I, J}^{\lambda}$, for each $(\mu, A, B) \in \mathcal{S}$, the set $\left\{Y_{A, B}^{\mu} \mid(\mu, A, B) \in \mathcal{S}\right\}$ is indeed a basis for $S_{q}(n, r)$.

\section{Codeterminants And Quantized enveloping Algebras}

In this section, we show that each of the factors $\xi_{I, I(\lambda)}$ and $\xi_{I(\lambda), J}$ in one of our codeterminants is equal, up to a power of $q$, to the product of the homomorphic image of $F_{S}$ in the quantized hyperalgebra (respectively $E_{T}$ ) and a particular idempotent in $S_{q}(n, r)$. Here $I=I_{R}(S)$ and $J=I_{R}(T)$, where $S$ and $T$ are $\lambda$ tableaux, and $F_{S}$ and $E_{T}$ are as defined in Section 4 . We use these results to give a quantum version of J. Green's description of the Carter-Lusztig basis theorem [8, Theorem 5.3b] at the end of the section. As well, the results in this section lay the groundwork for the main result in Section 7 which gives the expression of our basis elements as homomorphic images of explicit elements in the modified enveloping algebra.

The $n$-dimensional vector space $V_{\mathbb{Q}(q)}$ with basis $v_{1}, \ldots, v_{n}$ is a $U_{\mathbb{Q}(q)}$-module via

$$
E_{i} v_{k}=\delta_{i+1, k} v_{i}, \quad F_{i} v_{k}=\delta_{i k} v_{i+1}, \quad K_{j} v_{k}=q^{\delta_{j k}} v_{k}, \quad K_{j}^{-1} v_{k}=q^{-\delta_{j k}} v_{k}
$$

where $1 \leq i<n, 1 \leq j, k \leq n$. Let $V_{\mathcal{A}}$ be the $U_{\mathcal{A}}$-submodule of $V_{\mathbb{Q}(q)}$ generated by $v_{1}, \ldots, v_{n}$. The $r$-th tensor power, $V_{\mathcal{A}}^{\otimes r}$, is a $U_{\mathcal{A}}$-module via

$$
u\left(v_{i_{1}} \otimes v_{i_{2}} \otimes \cdots \otimes v_{i_{r}}\right)=\Delta^{r-1}(u)\left(v_{i_{1}} \otimes \cdots \otimes v_{i_{r}}\right),
$$

where $\Delta$ is defined in (3). Let $V^{\otimes r}$ denote the $U_{K}$-module obtained by base change. For $I=\left(i_{1}, i_{2}, \ldots, i_{r}\right) \in I(n, r)$, we let $v_{I}=v_{i_{1}} \otimes v_{i_{2}} \otimes \cdots \otimes v_{i_{r}}$. 
As well, $V^{\otimes r}$ is an $S_{q}(n, r)$-module via

$$
\xi v_{J}=\sum_{K \in I(n, r)}\left\langle x_{K, J}, \xi\right\rangle v_{K}, \xi \in S_{q}(n, r), v_{J} \in V^{\otimes r} .
$$

If $v_{J} \in V^{\otimes r}$, and $u \in U_{K}$ let $u v_{J}=\sum_{I \in I(n, r)} c_{I, J}(u) v_{I}$. We define a map $\theta: U_{K} \rightarrow$ $S_{q}(n, r)$ by

$$
\left\langle x_{I, J}, \theta(u)\right\rangle=c_{I, J}(u) .
$$

We will require the following two facts about the map $\theta$.

$$
\begin{gathered}
\theta(u) v_{I}=u v_{I}, u \in U_{K}, v_{I} \in V^{\otimes r}, \\
\theta\left(u_{1} u_{2}\right)=\theta\left(u_{1}\right) \theta\left(u_{2}\right), u_{1}, u_{2} \in U_{K} .
\end{gathered}
$$

The first statement above follows from the definition of $\theta$ and the second is proved in $[12$, Lemma 5.1].

For the next lemma, if $I=\left(i_{1}, \ldots, i_{r}\right), J=\left(j_{1}, \ldots, j_{r}\right) \in I(n, r)$, define $S_{1}(I, J)$ to be the number of pairs $\left(i_{a}, i_{b}\right)$ for which $i_{a}=i_{b}, i_{a}$ sits left of $i_{b}$ and the corresponding entries $j_{a}, j_{b}$ in $J$ satisfy $j_{a}>j_{b}$. Define $S_{2}(I, J)=S_{1}(J, I)$ to be the number of pairs $\left(j_{a}, j_{b}\right)$ for which $j_{a}=j_{b}, j_{a}$ sits left of $j_{b}$ and the corresponding entries $i_{a}$ and $i_{b}$ in $I$ are such that $i_{a}>i_{b}$. Let $\epsilon(I, J)=S_{1}(I, J)+S_{2}(I, J)$. The following lemma is a consequence of the relations (1). (A version of this is also given in [4, Lemma 1.1.4], who use somewhat different relations.)

Lemma 14. Let $I, J \in I(n, r)$. Then

$$
x_{I, J}=q^{\epsilon(I, J)} x_{(I, J)_{0}}+\text { terms involving } x_{S, T},
$$

where $(S, T)_{0}=(S, T),(S, T)>(I, J)_{0}$, and $(S, T)$ is not in the $S_{r}$-orbit of $(I, J)$.

We have the following consequences.

$$
\text { If }(I, J) \in \mathcal{I}^{2}(n, r) \text { and }\left\langle x_{P, Q}, \xi_{I, J}\right\rangle \neq 0 \text { then }(P, Q)_{0} \leq(I, J) \text {. }
$$

$$
\text { If }(I, J) \in \mathcal{I}^{2}(n, r) \text { then }\left\langle x_{I, Q}, \xi_{I, J}\right\rangle=0 \text { unless }(I, J) \sim(I, Q) .
$$

Note that (8) follows from the fact that, in this case, $I$ is weakly increasing so $x_{I, Q}=q^{\epsilon(I, Q)} x_{I, Q \sigma}$ where $I \sigma=I$. We will also require the following lemma [12, Lemma 5.3] in the proof of Lemma 16.

Lemma 15. Suppose that $Q=(\ldots, x, \ldots, y, \ldots)$ and $P=(\ldots, i, \ldots, x, \ldots)$, where $i<x<y \leq j$ and the $x$ and $y$ displayed in $Q$ are in the same positions as the $i$ and $x$ displayed in $P$. Then the coefficient of $v_{P}$ in $E_{i j} v_{Q}$ is 0 .

Lemma 16. Suppose that $T$ is standard, and let $J=I_{R}(T)$. Then

$$
\xi_{I(\lambda), I(\lambda)} \theta\left(E_{T}\right)=c \xi_{I(\lambda), J}
$$

for some $c \in \mathbb{Q}(q)$. 
Proof. The proof is by induction on the number of entries in $T$ which are different than the entries in $T(\lambda)$. If $T=T(\lambda)$ then $E_{T}$ is the identity, and $\xi_{I(\lambda), I(\lambda)} \theta\left(E_{T}\right)=$ $\xi_{I(\lambda), I(\lambda)}$.

Suppose that $T \neq T(\lambda)$, and suppose that the entries in the first row of $T$ are not all 1. (The argument is similar if the the first difference between $T$ and $T(\lambda)$ occurs in a lower row than the first.) Suppose that the first entry of $T$ in the first row that is not equal to 1 is $j$, and let $T^{\prime}$ be the tableau obtained by changing this $j$ to a 1 . Then $E_{T}=a E_{T^{\prime}} E_{1 j}$ for some $a \in \mathbb{Q}(q)$. Let $J^{\prime}$ be the sequence with $J^{\prime}=I_{R}\left(T^{\prime}\right)$. By induction we assume that

$$
\xi_{I(\lambda), I(\lambda)} \theta\left(E_{T^{\prime}}\right)=d \xi_{I(\lambda), J^{\prime}}, \quad \text { where } d \in \mathbb{Q}(q) .
$$

Write $\xi_{I(\lambda), I(\lambda)} \theta\left(E_{T}\right)$ as a linear combination of basis elements:

$$
\xi_{I(\lambda), I(\lambda)} \theta\left(E_{T}\right)=\sum_{(I(\lambda), Q) \in \mathcal{I}^{2}(n, r)} a_{Q} \xi_{I(\lambda), Q}, \quad a_{Q} \in \mathbb{Q}(q) .
$$

Then

$$
a_{Q}=\left\langle x_{I(\lambda), Q}, \xi_{I(\lambda), I(\lambda)} \theta\left(E_{T}\right)\right\rangle=\sum_{K \in I(n, r)}\left\langle x_{I(\lambda), K}, \xi_{I(\lambda), I(\lambda)}\right\rangle\left\langle x_{K, Q}, \theta\left(E_{T}\right)\right\rangle .
$$

By $(8),\left\langle x_{I(\lambda), K}, \xi_{I(\lambda), I(\lambda)}\right\rangle=0$ unless $(I(\lambda), I(\lambda)) \sim(I(\lambda), K)$, in which case $K=$ $I(\lambda)$. Thus $a_{Q}=\left\langle x_{I(\lambda), Q}, \theta\left(E_{T}\right)\right\rangle$, which is the coefficient of $v_{I(\lambda)}$ in $E_{T} v_{Q}$.

Suppose that $a_{Q} \neq 0$. We shall show that $Q=J$; if so, then $\xi_{I(\lambda), I(\lambda)} \theta\left(E_{T}\right)=$ $a_{Q} \xi_{I(\lambda), J}$ as desired. Suppose that $Q=I_{R}(S)$. Since $(I(\lambda), Q)$ is in $\mathcal{I}^{2}(n, r)$, the rows of $S$ are weakly increasing. We have

$$
\begin{aligned}
\xi_{I(\lambda), I(\lambda)} E_{T} v_{Q} & =\xi_{I(\lambda), I(\lambda)} \theta\left(E_{T}\right) v_{Q} \\
& =d \xi_{I(\lambda), J^{\prime}} \theta\left(E_{1 j}\right) v_{Q} \\
& =d \xi_{I(\lambda), J^{\prime}} E_{1 j} v_{Q} .
\end{aligned}
$$

Suppose that $E_{1 j} v_{Q}=\sum_{R} c_{R} v_{R}$, where each $c_{R} \in \mathbb{Q}(q)$. Then

$$
d \xi_{I(\lambda), J^{\prime}} E_{1 j} v_{Q}=\sum_{R} d c_{R} \xi_{I(\lambda), J^{\prime}} v_{R}
$$

Suppose, for some $R$, that

$$
c_{R} \xi_{I(\lambda), J^{\prime}} v_{R}=c_{R} \sum_{K \in I(n, r)}\left\langle x_{K, R}, \xi_{I(\lambda), J^{\prime}}\right\rangle v_{K} \neq 0 .
$$

Then by $(7),(K, R)_{0} \leq\left(I(\lambda), J^{\prime}\right)$. Since $\left(I(\lambda), J^{\prime}\right)$ is smallest in its orbit, $(K, R)_{0}=$ $\left(I(\lambda), J^{\prime}\right)$. Thus $K=I(\lambda) \sigma$ and, in particular, $R=J^{\prime} \sigma$ for some $\sigma \in R(\lambda)$. So after applying $E_{1 j}$ to $v_{Q}$, we get a linear combination of terms, one of which is $v_{J^{\prime} \sigma}$. Thus $Q$ has one more $j$ than $J^{\prime}$, and one less 1 . One possibility is that $Q$ is the same as $J^{\prime} \sigma$ except that a $j$ has been replaced by a 1 . Another possibility, if $j>2$, is that when applying $E_{1 j}$ to $Q$, a $y$ in $Q$ is changed to an $x$, and an $x$ in $Q$ is changed to a 1 , where $1<x<y \leq j$. Here $J^{\prime} \sigma$, and $Q$ have the form

$$
\begin{aligned}
J^{\prime} \sigma & =(1, \ldots, 1, \ldots, x, \ldots) \\
Q & =(1, \ldots, x, \ldots, y, \ldots)
\end{aligned}
$$


But from Lemma 15, in the latter case the coefficient of $v_{J^{\prime} \sigma}$ in $E_{i j} v_{Q}$ is 0 . Since we are assuming that $c_{R} \xi_{I(\lambda), J^{\prime}} v_{R} \neq 0$, it follows that $Q$ is the same as $J^{\prime} \sigma$ after replacing a $j$ by a 1 .

Since $T^{\prime}$ has no 1's below the first row, and $\sigma \in R(\lambda)$, the $j$ in $Q$ which is changed to a 1 in $J^{\prime} \sigma$ occurs in the first row of $S$. Since the first row of $S$ is weakly increasing, $J^{\prime} \sigma$ and $Q$ have the form

$$
\begin{aligned}
J^{\prime} \sigma & =(1, \ldots, j, j, \ldots, 1, \ldots, j, \ldots) \\
Q & =(1, \ldots, j, j, \ldots, j, \ldots, j, \ldots) .
\end{aligned}
$$

Now the first $\lambda_{1}$ entries of $J^{\prime}$ are weakly increasing, so $\sigma$ must switch the 1 among the middle of the $j$ 's to the front of $J^{\prime}$. So $J^{\prime}$ looks like $Q$ except that the first $j$ in $Q$ is changed to a 1 in $J^{\prime}$. Since $Q$ is identical to $J^{\prime}$ except that the first $j$ in $Q$ has been changed to a 1 , it follows that $Q=J$, and the proof is complete.

Lemma 17. Let $T$ be standard with $J=I_{C}(T)$. Then $\left\langle x_{I_{C}(\lambda)}, \theta\left(E_{T}\right)\right\rangle=1$.

Proof. In [12, Proposition 5.1], it is proved that the $K$-linear map $\phi: V^{\otimes r} \rightarrow \nabla_{q}(\lambda)$ defined by $\phi\left(v_{I}\right)=X_{I_{C}(\lambda), I}^{\lambda}$ is a $U_{K^{-}}$-epimorphism. In [16, Theorem 4.8], it is proved that $E_{T_{J}} X_{I_{C}(\lambda), J}^{\lambda}=X_{I_{C}(\lambda), I_{C}(\lambda)}^{\lambda}$.

Suppose that $E_{T_{J}} v_{J}=\sum_{I} a_{I} v_{I}$. Then $X_{I_{C}(\lambda), I_{C}(\lambda)}^{\lambda}=E_{T_{J}} X_{I_{C}(\lambda), J}^{\lambda}=\phi\left(E_{T_{J}} v_{J}\right)=$ $\phi\left(\sum_{I} a_{I} v_{I}\right)=\sum a_{I} \phi\left(v_{I}\right)=\sum a_{I} X_{I_{C}(\lambda), I}^{\lambda}$. Thus $a_{I_{C}(\lambda)}=1$.

Theorem 18. Let $T$ be standard with $J=I_{C}(T)$ and $J^{\prime}=I_{R}(T)$. Then

$$
\xi_{I(\lambda), I(\lambda)} \theta\left(E_{T}\right)=q^{-s(T)} \xi_{I(\lambda), J^{\prime}} .
$$

Proof. From Lemma 16, we have $\xi_{I(\lambda), I(\lambda)} \theta\left(E_{T}\right)=c \xi_{I(\lambda), J^{\prime}}$. Thus

$$
\left\langle x_{I_{C}(\lambda), J}, \xi_{I(\lambda), I(\lambda)} \theta\left(E_{T}\right)\right\rangle=\left\langle x_{I_{C}(\lambda), J}, c \xi_{I(\lambda), J^{\prime}}\right\rangle .
$$

The left hand side of this equation is equal to

$$
\left\langle x_{I_{C}(\lambda), J}, \xi_{I(\lambda), I(\lambda)} \theta\left(E_{T}\right)\right\rangle=\sum_{K}\left\langle x_{I_{C}(\lambda), K}, \xi_{I(\lambda), I(\lambda)}\right\rangle\left\langle x_{K, J}, \theta\left(E_{T}\right)\right\rangle .
$$

$\operatorname{By}(7),\left\langle x_{I_{C}(\lambda), K}, \xi_{I(\lambda), I(\lambda)}\right\rangle=0$ unless $\left(I_{C}(\lambda), K\right)_{0} \leq(I(\lambda), I(\lambda))$. Since the pair $(I(\lambda), I(\lambda))$ is smallest in its orbit, $\left(I_{C}(\lambda), K\right)_{0}=(I(\lambda), I(\lambda))$. Thus $K=I_{C}(\lambda)$ and

$$
\begin{aligned}
\left\langle x_{I_{C}(\lambda), J}, \xi_{I(\lambda), I(\lambda)} \theta\left(E_{T}\right)\right\rangle & =\left\langle x_{I_{C}(\lambda), I_{C}(\lambda)}, \xi_{I(\lambda), I(\lambda)}\right\rangle\left\langle x_{I_{C}(\lambda), J}, \theta\left(E_{T}\right)\right\rangle \\
& =q^{\epsilon\left(I_{C}(\lambda), I_{C}(\lambda)\right)}\left\langle x_{I_{C}(\lambda), J}, \theta\left(E_{T}\right)\right\rangle \text { by Lemma } 14 \\
& =\left\langle x_{I_{C}(\lambda), J}, \theta\left(E_{T}\right)\right\rangle=1, \text { by Lemma } 17 .
\end{aligned}
$$

It follows that $\left\langle x_{I_{C}(\lambda), J}, c \xi_{I(\lambda), J^{\prime}}\right\rangle=1$.

Now $\left(I_{C}(\lambda), J\right)$ and $\left(I(\lambda), J^{\prime}\right)$ belong to the same orbit and $\left(I(\lambda), J^{\prime}\right)$ is the smallest in this orbit so $\left(I_{C}(\lambda), J\right)_{0}=\left(I(\lambda), J^{\prime}\right)$. Thus, by Lemma 14 ,

$$
\begin{aligned}
\left\langle x_{I_{C}(\lambda), J}, \xi_{I(\lambda), J^{\prime}}\right\rangle & =\left\langle q^{\epsilon\left(I_{C}(\lambda), J\right)} x_{\left(I_{C}(\lambda), J\right)_{0}}, \xi_{I(\lambda), J^{\prime}}\right\rangle \\
& =\left\langle q^{\epsilon\left(I_{C}(\lambda), J\right)} x_{I(\lambda), J^{\prime}}, \xi_{I(\lambda), J^{\prime}}\right\rangle \\
& =q^{\epsilon\left(I_{C}(\lambda), J\right)} .
\end{aligned}
$$

In $\left[12\right.$, Lemma 5.8] it was proved that $\epsilon\left(I_{C}(\lambda), J\right)=s(T)$. Indeed, $S_{1}\left(I_{C}(\lambda), J\right)$ is equal to the number of pairs $\left(i_{a}, i_{b}\right)$ in $I_{C}(\lambda)$ for which $a<b, i_{a}=i_{b}$ and $j_{a}>j_{b}$. 
But, if $i_{a}=i_{b}$, this means that the corresponding entries $j_{a}$ and $j_{b}$ are in the same row. Since $j_{a}$ sits left of $j_{b}$ and $T$ is standard, $j_{a} \leq j_{b}$, from which it follows that $S_{1}\left(I_{C}(\lambda), J\right)=0$. Now, $S_{2}\left(I_{C}(\lambda), J\right)$ is equal to the number of pairs $\left(j_{a}, j_{b}\right)$ in $J$ with $a<b, j_{a}=j_{b}$ and $i_{a}>i_{b}$. If $a<b$, then $j_{a}$ sits in a column to the left of $j_{b}$. If $i_{a}>i_{b}$, then $j_{a}$ sits in a row below $j_{b}$. Thus $S_{2}\left(I_{C}(\lambda), J\right)=s(T)=\epsilon\left(I_{C}(\lambda), J\right)$. It follows that $c=q^{-s(T)}$.

Given $I=\left(i_{1}, i_{2}, \ldots, i_{r}\right) \in I(n, r)$, define $\beta(I)$ to be the number of pairs $(a, b)$ for which $a<b$ and $i_{a} \neq i_{b}$. For example, if $I=(1,4,1,3,3)$, then $\beta(I)=8$. In [16], we defined a bilinear form $():, V^{\otimes r} \times V^{\otimes r} \rightarrow \mathbb{Q}(q)$ by

$$
\left(v_{I}, v_{J}\right)=q^{\beta(I)} \delta_{I J}
$$

We have an antiautomorphism $\tau: U_{K} \rightarrow U_{K}$ defined by

$$
\tau\left(E_{i}\right)=F_{i}, \quad \tau\left(F_{i}\right)=E_{i}, \quad \tau\left(K_{i}\right)=K_{i} .
$$

In [16, Theorem 5.2] it was shown that the form above is a $U_{K}$-contravariant form; that is

$$
(u v, w)=(v, \tau(u) w), u \in U_{K}, v, w \in V^{\otimes r} .
$$

Since $\left(f_{i j} v, w\right)=\left(v, e_{i j} w\right)$ for $1 \leq i, j<n$ and $v, w \in V$, we have

$$
\left(F_{T} v, w\right)=\left(v, E_{T} w\right), v, w \in V^{\otimes r}, T \text { standard. }
$$

Given a standard $\lambda$-tableau $T$ with $k$ rows, define $r_{i}(T)$ to be the number of pairs $(a, b)$ in row $i$ of $T$ in which $a$ is in a box to the left of $b$ and $a \neq b$ (since $T$ is standard, it must be that $a<b)$. Define $r(T)=\sum_{i=1}^{k} r_{i}(T)$.

Example. If $T=$\begin{tabular}{|l|l|l|l|}
\hline 1 & 2 & 3 & 3 \\
\hline 4 & 5 &
\end{tabular} then $r(T)=6$.

Theorem 19. Let $S$ be a standard $\lambda$-tableau with $I^{\prime}=I_{R}(S)$. Then $\theta\left(F_{S}\right) \xi_{I(\lambda), I(\lambda)}=$ $q^{-r(S)} \xi_{I^{\prime}, I(\lambda)}$.

Proof. In Theorem 18, we proved that $\xi_{I(\lambda), I(\lambda)} \theta\left(E_{S}\right)=q^{-s(S)} \xi_{I(\lambda), I^{\prime}}$. Thus, if

$$
\xi_{I(\lambda), I(\lambda)} \theta\left(E_{S}\right)=\sum_{(I(\lambda), Q) \in \mathcal{I}^{2}(n, r)} b_{I(\lambda), Q} \xi_{I(\lambda), Q},
$$

then $b_{I(\lambda), Q}=0$ unless $Q=I^{\prime}$. In other words, if $(I(\lambda), Q) \in \mathcal{I}^{2}(n, r)$, then the coefficient of $v_{I(\lambda)}$ in $E_{S} v_{Q}$ is zero unless $Q=I^{\prime}$. Furthermore, the coefficient of $v_{I(\lambda)}$ in $E_{S} v_{I^{\prime}}$ is $q^{-s(S)}$.

For every $(A, B) \in \mathcal{I}^{2}(n, r)$ such that $B \sim I(\lambda)$, there exists $Q \in I(n, r)$ with $(I(\lambda), Q) \in \mathcal{I}^{2}(n, r)$ such that $(A, B)=(Q, I(\lambda))_{0}$. Thus, by Lemma 2 ,

$$
\theta\left(F_{S}\right) \xi_{I(\lambda), I(\lambda)}=\sum_{(I(\lambda), Q) \in \mathcal{I}^{2}(n, r)} a_{Q, I(\lambda)} \xi_{(Q, I(\lambda))_{0}}=\sum_{(I(\lambda), Q) \in \mathcal{I}^{2}(n, r)} a_{Q, I(\lambda)} \xi_{Q, I(\lambda)},
$$

where $a_{Q, I(\lambda)}$ is the coefficient of $v_{Q}$ in $F_{S} v_{I(\lambda)}$. For any $Q$ with $(I(\lambda), Q) \in \mathcal{I}^{2}(n, r)$, we have

On the other hand,

$$
\left(F_{S} v_{I(\lambda)}, v_{Q}\right)=\left(a_{Q, I(\lambda)} v_{Q}, v_{Q}\right)=q^{\beta(Q)} a_{Q, I(\lambda)} .
$$

$$
\left(F_{S} v_{I(\lambda)}, v_{Q}\right)=\left(v_{I(\lambda)}, E_{S} v_{Q}\right)
$$


Thus

$$
q^{\beta(Q)} a_{Q, I(\lambda)}=\left(v_{I(\lambda)}, E_{S} v_{Q}\right) .
$$

As mentioned earlier, if $Q \neq I^{\prime}$, then the coefficient of $v_{I(\lambda)}$ in $E_{S} v_{Q}$ is zero so, from the above equation, we have $a_{Q, I(\lambda)}=0$ unless $Q=I^{\prime}$.

If $Q=I^{\prime}$, then

$$
q^{\beta\left(I^{\prime}\right)} a_{I^{\prime}, I(\lambda)}=\left(v_{I(\lambda)}, E_{S} v_{I^{\prime}}\right)=\left(v_{I(\lambda)}, q^{-s(S)} v_{I(\lambda)}\right)=q^{\beta(I(\lambda))} q^{-s(S)},
$$

so that $a_{I^{\prime}, I(\lambda)}=q^{\beta(I(\lambda))-s(S)-\beta\left(I^{\prime}\right)}$.

The number $\beta\left(I^{\prime}\right)$ is equal to the number of pairs $(a, b)$ in $S, a \neq b$, with $a$ and $b$ in the same row but $a$ sitting to the left of $b$ plus the number of pairs $(a, b)$ in $S$ with $a \neq b$ and $b$ in a row below $a$. Since $\beta(I(\lambda))$ counts all pairs $(a, b)$ in $S$ with $b$ in a row below $a$, including those pairs where $a=b$, we have

$$
\beta(I(\lambda))=\beta\left(I^{\prime}\right)+s(S)-r(S) .
$$

Thus $a_{I^{\prime}, I(\lambda)}=q^{-r(S)}$ and, indeed, $\theta\left(F_{S}\right) \xi_{I(\lambda), I(\lambda)}=q^{-r(S)} \xi_{I^{\prime}, I(\lambda)}$.

Define $z_{\lambda}=\sum_{\sigma \in C(\lambda)}(-q)^{-\ell(\sigma)} v_{I_{C}(\lambda) \sigma} \in V_{\mathcal{A}}^{\otimes r}$, where $\ell(\sigma)$ denotes the length of the permutation $\sigma$. Let $\Delta(\lambda)_{\mathcal{A}}$ denote the $U_{\mathcal{A}}$-submodule of $V_{\mathcal{A}}^{\otimes r}$ generated by $z_{\lambda}$. The $q$-Weyl module for the hyperalgebra $U_{K}$ is defined by base change; $\Delta_{q}(\lambda)=\Delta(\lambda)_{\mathcal{A}} \otimes K$.

In $[16, \S 5]$, a quantum version of the Carter-Lusztig basis theorem was given. In particular, it was proved that the set

$$
\left\{F_{T} z_{\lambda} \mid T \text { is standard }\right\}
$$

is a $K$-basis for $\Delta_{q}(\lambda)$.

Let

$$
\mathcal{J}_{\lambda}=\left\{J \in I(n, r) \mid J=I_{R}(T) \text { where } T \text { is a standard } \lambda \text {-tableau }\right\}
$$

and, if $J=I_{R}(T)$, define $r(J)=r(T)$, where $r(T)$ is as defined above Theorem 19 . Theorem 19 allows us to give the quantum version of the Carter-Lusztig basis in terms of elements in the $q$-Schur algebra. The following corollary is the quantum version of [8, Theorem 5.3b].

Corollary 20. The set

$$
\left\{\xi_{J, I(\lambda)} z_{\lambda} \mid J \in \mathcal{J}_{\lambda}\right\}
$$

is a $K$-basis for $\Delta_{q}(\lambda)$.

Proof. First note that $\xi_{I(\lambda), I(\lambda)} z_{\lambda}=z_{\lambda}$. Indeed, if $\sigma \in C(\lambda)$, then

$$
\xi_{I(\lambda), I(\lambda)} v_{I_{C}(\lambda) \sigma}=\sum_{M}\left\langle x_{I_{C}(\lambda), M}, \xi_{I(\lambda), I(\lambda)}\right\rangle v_{M}
$$

and $\left\langle x_{I_{C}(\lambda), M}, \xi_{I(\lambda), I(\lambda)}\right\rangle=0$ unless $\left(I_{C}(\lambda) \sigma, M\right)_{0} \leq(I(\lambda), I(\lambda))$. Since $(I(\lambda), I(\lambda))$ is smallest in its orbit, $\left(I_{C}(\lambda) \sigma, M\right)_{0}=(I(\lambda), I(\lambda))$. Thus $M=I_{C}(\lambda) \sigma$ and so $\xi_{I(\lambda), I(\lambda)} v_{I_{C}(\lambda) \sigma}=v_{I_{C}(\lambda) \sigma}$.

Now, if $T$ is standard with $J=I_{R}(T)$, then

$$
F_{T} z_{\lambda}=\theta\left(F_{T}\right) z_{\lambda}=\theta\left(F_{T}\right) \xi_{I(\lambda), I(\lambda)} z_{\lambda}=q^{-r(J)} \xi_{J, I(\lambda)} z_{\lambda},
$$

and the Corollary now follows. 


\section{Codeterminants AND MOdified QUANTIZED EnVEloping AlgeBras}

Doty [5] observes that the $q$-Schur algebra $S_{q}(n, r)$ is isomorphic to a homomorphic image of Lusztig's modified quantized enveloping algebra $\dot{U}$. (A version of this was proved earlier in [1], who did not use the notation $\dot{U}$.) The $\mathbb{Q}(q)$-algebra $\dot{U}\left[13\right.$, Chap. 23] is obtained from the triangular decomposition $U_{\mathbb{Q}(q)}=U^{-} U^{0} U^{+}$ by replacing $U^{0}$ by a direct sum of infinitely many 1-dimensional algebras, each generated by an idempotent $1_{\lambda}$, one for each weight $\lambda$.

Weights $\lambda$ can be identified with compositions of $r$ into at most $n$ parts, that is, $\lambda=\left(\lambda_{1}, \ldots, \lambda_{n}\right)$ where each $\lambda_{i}$ is a non-negative integer, and $\sum_{i} \lambda_{i}=r$. The weight $\lambda$ is dominant precisely when $\lambda$ is a partition, that is, $\lambda_{1} \geq \lambda_{2} \geq \cdots \geq \lambda_{n}$. For a weight $\lambda$, we have the sequence $I(\lambda)$ which consists of $\lambda_{1} 1$ 's, then $\lambda_{2} 2$ 's, etc. This agrees with our earlier version of $I(\lambda)$, defined when $\lambda$ is a partition.

Lemma 21. For a composition $\lambda$, the element $\xi_{I(\lambda), I(\lambda)}$ is an idempotent.

Proof. Take $(I, J) \in \mathcal{I}^{2}(n, r)$. We have

$$
\left\langle x_{I, J}, \xi_{I(\lambda), I(\lambda)} \xi_{I(\lambda), I(\lambda)}\right\rangle=\sum_{K}\left\langle x_{I, K}, \xi_{I(\lambda), I(\lambda)}\right\rangle\left\langle x_{K, J}, \xi_{I(\lambda), I(\lambda)}\right\rangle
$$

Note that since $(I, J) \in \mathcal{I}^{2}(n, r)$, then $\left\langle x_{I, K}, \xi_{I(\lambda), I(\lambda)}\right\rangle \neq 0$ only when $I=I(\lambda)$, and then only when $K=I(\lambda)$, and similarly $\left\langle x_{K, J}, \xi_{I(\lambda), I(\lambda)}\right\rangle \neq 0$ only when $K=J=I(\lambda)$. So $\left\langle x_{I, J}, \xi_{I(\lambda), I(\lambda)} \xi_{I(\lambda), I(\lambda)}\right\rangle=0$ unless $I=J=I(\lambda)$ and $\left\langle x_{I(\lambda), I(\lambda)}, \xi_{I(\lambda), I(\lambda)} \xi_{I(\lambda), I(\lambda)}\right\rangle=1$. Therefore $\xi_{I(\lambda), I(\lambda)} \xi_{I(\lambda), I(\lambda)}=\xi_{I(\lambda), I(\lambda)}$.

From [13, Chap. 23], the elements of $\dot{U}$ can be written as $x^{-} 1_{\lambda} x^{+}$, and also as $y^{+} 1_{\lambda} y^{-}$, where $x^{-}, y^{-} \in U^{-}, x^{+}, y^{+} \in U^{+}$. Relations for these elements are given in Section 23.1.3 of [13].

Lusztig [13] also defines an $\mathcal{A}$-form $\dot{U}_{\mathcal{A}}$ of $\dot{U}$, generated by $E_{i}^{(a)}, F_{i}^{(a)}$, and the idempotents $1_{\lambda}$. As in [13, Chap. 31], for a field $K$ containing a non-zero element $q$,

$$
\dot{U}_{K}=K \otimes_{\mathcal{A}} \dot{U}_{\mathcal{A}}
$$

For an element $u$ of $\in \dot{U}_{\mathcal{A}}$, we also denote by $u$ the element $1 \otimes u \in \dot{U}_{K}$.

We give the homomorphism $\dot{\theta}: \dot{U}_{K} \rightarrow S_{q}(n, r)$ explicitly as follows:

$$
\dot{\theta}\left(x^{-} 1_{\lambda} x^{+}\right)=\theta\left(x^{-}\right) \xi_{I(\lambda), I(\lambda)} \theta\left(x^{+}\right), \quad x^{-} \in U_{K}^{-}, x^{+} \in U_{K}^{+} .
$$

That this is a homomorphism follows from the fact that $\theta$ is a homomorphism, from the previous Lemma, and from Lusztig's relations.

We now have

Theorem 22. Let $\lambda$ be a partition and let $S, T$ be standard $\lambda$-tableaux. Let $I=$ $I_{R}(S)$ and $J=J_{R}(T)$. Then the codeterminant $\xi_{I, I(\lambda)} \xi_{I(\lambda), J}$ is given by

$$
\xi_{I, I(\lambda)} \xi_{I(\lambda), J}=q^{r(S)+s(T)} \dot{\theta}\left(F_{S} 1_{\lambda} E_{T}\right) .
$$

Proof. This follows from Theorems 18 and 19. 


\section{REFERENCES}

[1] A. A. Beilinson, G. Lusztig, and R. MacPherson, A geometric setting for the quantum deformation of $G L_{n}$, Duke Math. J. 61 (1990), 655-677.

[2] R. Dipper and G. James, The q-Schur algebras, Proc. London Math. Soc. 59 (1989), 23-50.

[3] _ q-tensor space and q-Weyl modules, Trans. Amer. Math. Soc. 327 (1991), 252-282.

[4] R. Dipper and S. Donkin, Quantum $G L_{n}$, Proc. London Math. Soc. (3) 63 (1991), 165-211.

[5] S. Doty, Presenting generalized q-Schur algebras, Represent. Theory 7 (2003), 196-213.

[6] J. Du, The modular representation theory of q-Schur algebras, Trans. Amer. Math. Soc. 329 (1992), 253-271.

[7] J. A. Green, Combinatorics and the Schur algebra, J. Pure Applied Algebra 88 (1993), 89-106.

[8] _ Polynomial Representations of $G L_{n}$, Lecture Notes in Math., vol. 830, Springer, Berlin/Heidelberg/New York, 1980.

[9] R. M. Green, A straightening formula for quantized codeterminants, Comm. Algebra 24 (1996), 2887-2913.

[10] J. Hu, A combinatorial approach to representations of quantum linear groups, Comm. Algebra 26 (1998), 2591-2621.

[11] R. Q. Huang and J. J. Zhang, Standard basis theorem for quantum linear groups, Adv. Math. 102 (1993), 202-229.

[12] D. Janzen, S. Phillips, and A. Stokke, A combinatorial description of the quantum Désarménien matrix via the q-Schur algebra, J. Algebra 304 (2006), 906-926.

[13] G. Lusztig, Introduction to Quantum Groups, Birkhaüser, Boston, 1993.

[14] B. Parshall and J.-p. Wang, Quantum Linear Groups, Memoirs Amer. Math. Soc., vol. 439, Amer. Math. Soc., Providence, 1991.

[15] Yu. I. Manin, Quantum Groups and Noncommutative Geometry, Publications du Centre de Récherches Mathématiques, Université de Montréal, Montreal, 1988.

[16] A. Stokke, A quantum version of the Désarménien matrix, J. Alg. Combinatorics 22 (2005), 303-316.

[17] E. Taft and J. Towber, Quantum deformation of flag schemes and Grassman schemes I-a q-deformation of the shape algebra for $G L(n)$, J. Algebra 142 (1991), 1-36.

[18] D. Woodcock, Straightening codeterminants, J. Pure Applied Algebra 88 (1993), 317-320.

University of Alberta, Department of Mathematical and Statistical Sciences, Edmonton, Alberta, Canada T6G 2G1

E-mail address: gcliff@math.ualberta.ca

University of Winnipeg, Department of Mathematics and Statistics, Winnipeg, Manitoba, Canada R3B 2E9

E-mail address: a.stokke@uwinnipeg.ca 